\title{
DIMENSIONES DE CONTENIDO DE PREOCUPACIÓN EN POBLACIÓN DE EDAD AVANZADA
}

\author{
ROBERTO NUEVO, MARÍA IZAL, IGNACIO MONTORIO, \\ ANDRÉS LOSADA y MARÍA MÁRQUEZ
}

\author{
Universidad Autónoma de Madrid
}

(Aceptado en febrero de 2003)

\begin{abstract}
Este estudio se dirige a analizar las principales dimensiones de contenidos de preocupación entre las personas mayores, partiendo de un amplio listado de items sobre contenidos específicos relevantes en esta edad. La muestra se compuso de 105 personas mayores de 65 años, seleccionadas mediante muestreo aleatorio a partir del censo de una localidad urbana. Mediante análisis factorial se retuvieron 35 items agrupados en cinco subescalas: Economía, Salud, Familia, Competencias Personales y aspectos Socio-Afectivos. Estas cinco subescalas tuvieron correlaciones positivas y significativas con medidas de preocupación, ansiedad y depresión. Las personas con niveles altos de preocupación obtuvieron puntuaciones significativamente superiores en todas las escalas. La Escala de Preocupaciones (EP) compuesta por los 35 items retenidos se propone como un instrumento fiable y válido de evaluación de los contenidos de preocupación en las personas mayores, aunque son precisas validaciones adicionales.
\end{abstract}

Palabras Clave: Preocupación, evaluación, personas mayores, trastornos de ansiedad.

\section{Dimensions of content of worry among older adults}

This study is aimed to analyze the main dimensions of contents of worry in the elderly, from the starting point of a broad pool of items referring to specific contents of worry relevant to this age group. The sample was composed by 105 adults aged 65 and over, who were randomly selected from the census of an urban locality. A Factor Analysis was used and 35 items, grouping into 5 subscales (Financial, Health, Family, Personal Competences, and Socio-Affective concerns) were retained. These five subscales correlated positive and significantly with worry, anxiety and depression measures. High worriers scored significantly higher in every subscale. The 35-item Worry Scale (Escala de Preocupaciones; EP), is proposed as a reliable and valid instrument to assess contents of worry among older adults, although additional validations are still required.

Key words: Worry, assessment, elderly people, anxiety disorders.

\section{INTRODUCCIÓN}

Durante las últimas dos décadas ha habido un interés progresivamente creciente en el estudio de la preocupación, sea como fenómeno normal y adaptativo, presente en alguna medida en todos los seres humanos, sea como un proceso clí-

Correspondencia: Ignacio Montorio, Departamento de Psicología Biológica y de la Salud, Facultad de Psicología, Universidad Autónoma de Madrid, Campus de Cantoblanco, Carretera de Colmenar Km. 15,300, 28049 Madrid. Teléfono:91 397 4060, 91397 5220, Fax: 91397 5215, Correo-e: ignacio.montorio@uam.es nico relevante en muchos trastornos emocionales, aunque especialmente vinculado al Trastorno de Ansiedad Generalizada (TAG), trastorno cuya principal característica diagnóstica según el DSMIV-TR (APA, 2001) es, precisamente, una preocupación excesiva y de difícil controlabilidad. Una de las dificultades vigente en la comprensión y conocimiento de la preocupación es la definición de los contenidos en los que la preocupación se centra y la importancia diferencial que los contenidos de preocupación pueden tener para distintas 
cohortes de edad en la génesis y el mantenimiento de la preocupación excesiva. La especificación de los contenidos implicados en la preocupación excesiva puede ser un dato relevante en la definición general del TAG como categoría diagnóstica y, particularmente, puede ayudar a definir lo que puede considerarse un trastorno en grupos específicos de población. La relevancia del estudio de los contenidos de preocupación queda reflejada en los planteamientos de M.W. Eysenck (1984), autor que postuló la existencia de conglomerados organizados de información relacionada con la preocupación en la memoria a largo plazo. Estos conglomerados reflejarían áreas de preocupación. Según Eysenck, el número y estructura de esos conglomerados de preocupación constituiría el determinante más importante de con qué frecuencia y duración un sujeto experimenta la preocupación. Así, las personas que tuvieran conglomerados de información relacionada con la preocupación altamente interrelacionados en su memoria a largo plazo accederían y activarían con mayor facilidad y rapidez elementos de preocupación y esquemas de amenaza, que a su vez actuarían sobre los procesos perceptivos produciendo interferencias y sesgos atencionales. La noción de que la información está agrupada de este modo en la memoria a largo plazo es consistente con diferentes planteamientos teóricos que reconocen la importancia del almacenamiento modular (p.ej., Bower, 1981). La importancia que tienen estos factores estructurales asociados a dominios específicos de preocupación en el inicio y mantenimiento de la preocupación ha sido comprobada experimentalmente (Pratt, Tallis y Eysenck, 1997; Tallis, Eysenck y Mathews, 1991).

La mayoría de la investigación existente sobre los contenidos de preocupación ha trabajado con muestras de adultos jóvenes, especialmente con estudiantes universitarios. En estos trabajos se han propuesto diversas clasificaciones de contenidos de preocupación que, lógicamente, se encuentran determinadas por aspectos que son considerados fundamentales para el mantenimiento de la satisfacción vital en los grupos de edad y en el contexto cultural estudiado. Así, las áreas más relevantes de preocupación encontradas se centran en las relaciones interpersonales, falta de confianza en sí mismo, falta de objetivos para el futuro, trabajo, temas económicos y temas sociopolíticos (Tallis, Eysenck y Mathews, 1992), aunque algunos autores han considerado que estas dimensiones básicas de preocupación podían integrarse en dos: relaciones sociales y salud física (Eysenck, 1992; Wells, 1994). No está claro, sin embargo, que los contenidos de la preocupación varíen en función del grado en el que una persona se preocupa, o incluso de la presencia de TAG. Aunque un supuesto básico de la mayoría de los estudios sobre contenidos es que las preocupaciones de los sujetos normales deberían diferenciarse de algún modo de las de los sujetos clínicos y, entre éstos, las de los pacientes de TAG deberían también poder diferenciarse claramente de los pacientes con otro trastorno de ansiedad, un trastorno del estado de ánimo u otro trastorno psicológico, muchos trabajos han propuesto que no existen en realidad tales diferencias o son muy escasas (p.ej. Craske, Rapee, Jackel y Barlow, 1989; Sanderson y Barlow, 1990). Así, Wells (1994) afirma que estos grupos no se diferencian sustancialmente en el contenido de sus preocupaciones, sino que "tienden a diferir más en términos de dimensiones de proceso tales como su controlabilidad».

Probablemente, estos resultados puedan deberse a una confusión terminológica. Es necesario diferenciar, por un lado, entre dominios generales de preocupación (las categorías generales como 
salud, economía, etc.) y preocupaciones específicas (p.ej., "tener un ataque al corazón o un cáncer") y, por otro, entre ambos aspectos referidos al contenido y la manera en la que se procesan estos contenidos. Frecuentemente se ha encontrado que las personas con o sin TAG, con o sin trastorno, con niveles altos o bajos de ansiedad-rasgo, se preocupan por los mismos temas generales, pero por diferentes contenidos específicos y, además, lo hacen de un modo distinto. Por ejemplo puede que existan diferencias en la cantidad de preocupaciones, pero no en el tipo de preocupaciones (que unas y otras personas se preocupen sobre temas de salud, pero las personas con preocupación patológica tengan o encuentren más temas de salud por los que preocuparse, aparte de que además lo hagan de un modo diferente). Así, con este enfoque, se han encontrado tres diferencias básicas en función de la presencia de TAG: 1) Los sujetos diagnosticados de TAG se preocupan sobre una mayor variedad de situaciones que los sujetos no clínicos (Craske et al., 1989); 2) También se preocupan más sobre cuestiones menores que los sujetos no clínicos (Shadick, Roemer, Hopkins, y Borkovec, 1991) o que sujetos con otro trastorno de ansiedad (Sanderson y Barlow, 1990); 3) Se preocupan más por el futuro (Dugas et al., 1998).

De todos modos, los resultados obtenidos con estas muestras de personas jóvenes no pueden generalizarse sin más a grúpos de población con trayectorias vitales y de cohorte muy diferentes, como son las personas mayores. Desafortunadamente, existen muy pocos trabajos que analicen las preocupaciones en la vejez. Y la mayor parte de los trabajos publicados que se han centrado en el análisis de los contenidos son de tipo descriptivo. Estos estudios han utilizado generalmente la «Worry Scale» (WS), una escala construida por Wisocki, Handen y
Morse (1986) para evaluar en personas mayores la frecuencia de las preocupaciones sobre tres contenidos generales: Economía, Salud y Relaciones Sociales. Los resultados con esta escala indican que las preocupaciones sociales son un factor de escasa relevancia en las personas mayores (p.ej., Wisocki, 1988), lo que vendría a indicar que, a diferencia de lo que ocurre en población general, donde la ansiedad tiene un fuerte componente socioevaluativo, en las personas mayores la ansiedad podría caracterizarse de otro modo. Adicionalmente, se ha encontrado usando esta misma escala (Skarborn y Nicki, 1996) y mediante entrevistas abiertas (Person y Borkovec, 1995; Powers, Wisocki y Whitbourne, 1992) que las preocupaciones principales de las personas mayores se centran en temas relacionados con la salud. De hecho, Person y Borkovec (1995), encontraron una relación lineal entre contenidos de preocupación y edad: las preocupaciones familiares, económicas y laborales disminuían con la edad, mientras las relacionadas con la salud aumentaban. Finalmente, dos estudios recientes indican diferencias en contenido entre personas mayores con o sin TAG, de tal forma que las preocupaciones más frecuentemente informadas por las personas mayores en general se relacionan con el bienestar de la familia, pero las preocupaciones por la salud son las que están más asociadas a la preocupación excesiva propia del TAG (Diefenbach, Stanley y Beck, 2001; Montorio, Nuevo, Márquez, Izal y Losada, 2003).

Respecto al modo de evaluar los contenidos de preocupación, se han utilizado dos tipos de medidas: los instrumentos estructurados, en los que se pregunta al sujeto por el grado o la frecuencia con la que se preocupa por un listado de contenidos específicos y prefijados; y los instrumentos de recuerdo libre, que preguntan abiertamente por los contenidos por los que se preocupa. En un estudio diri- 
gido a analizar las ventajas y problemas de ambos tipos de medida, Dugas, Freeston, Doucet, Lachance y Ladouceur (1995) concluyeron que debería recomendarse el uso de las medidas estructuradas siempre que éstas estén diseñadas para la evaluación de los contenidos en poblaciones específicas en las que se hayan fijado ya los contenidos más relevantes de preocupación. Los cuestionarios estructurados serían medidas reactivas que ofrecen un rango restringido de respuestas (Arnkoff y Glass, 1982) y llevan a los sujetos a realizar juicios dirigidos que favorecen respuestas genéricas y más estables frente a los instrumentos abiertos, que maximizan un patrón de respuesta ideográfico de forma no reactiva. Por lo tanto, el proceso de construcción de los instrumentos debería realizarse utilizando medidas abiertas para maximizar las posibilidades de no obviar contenidos relevantes. En esta línea, mediante un trabajo cualitativo con grupos de discusión de personas mayores, Wisocki, Hunt y Souza (no publicado; citado en Wisocki, 1994), propusieron un listado comprehensivo de 88 preocupaciones específicas que parecen relevantes en la vejez, aunque no ha sido seguido de un trabajo de reducción de la información del cuestionario ni se ha intentado emplearlo para proponer una escala manejable y que elimine la información redundante o superflua. Una validación al castellano de este listado de preocupaciones será, justamente, el punto de partida del trabajo que presentamos.

$\mathrm{El}$ presente estudio se dirige a analizar los contenidos de preocupación en una muestra de personas mayores españolas no institucionalizadas seleccionadas mediante muestreo aleatorio estratificado por edad y sexo a partir del censo de una localidad urbana. El objetivo del trabajo es doble. Primero, a partir de un amplio listado de items referidos a situaciones específicas que pueden preocupar a una persona mayor, se analizan las dimensiones de contenido en las que se agrupan las preocupaciones entre las personas mayores de nuestro contexto cultural. Mediante una progresiva reducción de la información redundante se pretende obtener una descripción de los factores básicos de la preocupación en la población estudiada. El listado de contenidos específicos agrupado por factores se presentará como punto de partida para una posible escala para la evaluación en las personas mayores españolas de los contenidos de preocupación, tanto en población general como entre las personas con patología emocional. Segundo, se pretende analizar la existencia de diferencias, en función de la gravedad de la preocupación, en los contenidos de preocupación resultantes de los primeros análisis, esto es, analizar si las personas que se preocupan con mayor frecuencia lo hacen además por contenidos generales diferentes.

\section{MÉTODO}

\section{Sujetos}

Mediante muestreo aleatorio estratificado proporcional (por edad y género) a partir del censo de Majadahonda, localidad urbana cercana a Madrid, fueron seleccionadas 195 personas mayores de 65 años, de las que 118 accedieron a participar en el estudio, aunque 13 de ellas fueron finalmente excluidas por existir sospechas de deterioro cognitivo en función de la puntuación en el MiniExamen Cognoscitivo (MEC, Folstein, Folstein y McHugh, 1975; versión española de Lobo, Ezquerra, Gómez, Sala y Seva, 1979). Así, la muestra final estuvo compuesta por 105 personas no institucionalizadas. El 39,8\% fueron hombres y el $60,2 \%$ mujeres y la edad media fue de $72,93(D T=5,74)$ con un rango entre 
62 y 90 años. Un $63,3 \%$ de las personas estaban casadas, un $33 \%$ viudas, el $2,8 \%$ solteras y el $0,9 \%$ separadas. El $33,9 \%$ de los sujetos no había realizado ningún estudio académico o los había interrumpido tempranamente, el 26,6\% había realizado estudios primarios, el $22 \%$ estudios secundarios y el $17,4 \%$ estudios superiores. Estas características son similares a las de la población mayor española, excepto el nivel educativo, que es superior en la muestra que compone el estudio, si bien entre las personas mayores es esperable que colaboren aquellas con mayor nivel educativo (Schaie, 1994).

\section{Variables}

Los contenidos de preocupación fueron evaluados inicialmente con una versión española de la versión de la Worry Scale for Older Adults-revised (WSOAR; Wisocki, 1994,; versión española de Montorio, Nuevo, Izal y Losada, 1998). La WSOA-R es una extensión de la WS (Worry Scale; Wisocki, Handen y Morse, 1986), un cuestionario de 35 items que evalúa la frecuencia de preocupaciones sobre tres contenidos: Economía, Salud y Aspectos Sociales. La versión revisada se compone de 88 items extraídos mediante el trabajo con grupos de discusión de personas mayores. Cubre seis dominios de preocupación: Economía, Salud, Aspectos Sociales, Aspectos Personales, Familia y Problemas Socio-políticos. En la adaptación española de la WSOA-R (Montorio et al., 1998) fueron eliminados cinco items que no eran aplicables a población española. Así, la versión empleada en este estudio se compone de 83 items con formato de respuesta tipo Likert (de 0, "nunca», a 4, "casi siempre»), todos ellos planteados en sentido positivo. La agrupación por subescalas es la siguiente: Economía (9 items), Salud (20), Aspectos Sociales (14), Aspectos Personales (18), Familia (15) y Problemas Socio-políticos (7). Además, se plantearon dos preguntas abiertas sobre posibles contenidos de preocupación, una antes de aplicar la WSOA-R y otra después, para comprobar la posible existencia de algún tema de preocupación que no estuviera incluido en el listado. Sin embargo, ninguna de las preocupaciones que emergieron durante las entrevistas aportaba algo original que no pudiera ser asimilado a alguno de los 83 elementos de la lista.

Otras variables fueron evaluadas con el fin de analizar la relación de los diferentes contenidos de preocupación con indicadorès de preocupación-rasgo, ansiedad-rasgo y depresión.

La preocupación-rasgo fue medida con una adaptación española del Inventario de Preocupación de Pensilvania (PSWQ, Penn State Worry Questionnaire; Meyer, Miller, Metzger y Borkovec, 1990; adaptación española de Nuevo, Montorio y Ruiz, 2002). El PSWQ es un instrumento de 16 items con formato de respuesta tipo Likert (rango $=1-5$ ) que evalúa una tendencia general a preocuparse o preocupación-rasgo. Ha demostrado buenas propiedades psicométricas, incluyendo una excelente capacidad para discriminar la preocupación patológica y excesiva característica del Trastorno de Ansiedad Generalizada, con población general (Fresco, Heimberg, Mennin y Turk, 2002; Ladouceur et al., 1992), con muestras clínicas de trastornos de ansiedad (Brown, Antony y Barlow, 1992) y de depresión (Starcevic, 1995) y con muestras de personas mayores en otros contextos geográficos (Beck, Stanley y Zebb, 1995) y españolas (Nuevo, Montorio y Ruiz, 2002).

La ansiedad-rasgo fue evaluada mediante la subescala Rasgo del Inventario de Ansiedad Estado-Rasgo (STAI-R, State-Trait Anxiety Inventory; Spielberger, 
Gorsuch y Lushene, 1970). La versión española de esta prueba (TEA, 1982) consta de 20 items tipo Likert (de 0, "nada» a 3, «mucho»), 7 de ellos invertidos, que evalúan el grado en el que la persona considera que distintas afirmaciones sobre sus emociones se ajustan a cómo se siente en general, en la mayoría de las ocasiones.

La gravedad de la depresión fue evaluada mediante la Escala de Depresión Geriátrica (GDS, Geriatric Depresión Scale; Brink et al., 1982), instrumento construido para su aplicación con personas mayores que presenta 30 items dicotómi$\cos (\mathrm{S} i ́ /$ No) sobre el modo en el que la persona se ha sentido durante la última semana. La versión española de la GDS (Izal y Montorio, 1996) presenta buenas propiedades psicométricas y elevadas sensibilidad y especificidad para identificar la presencia de Depresión Mayor (Montorio e Izal, 1996).

\section{Procedimiento}

A las personas seleccionadas a partir del censo se les envió una carta comunicándoles que el Ayuntamiento estaba recabando información sobre las preocupaciones de las personas mayores de la localidad. Posteriormente se contactó telefónicamente con ellas para concretar una cita para administrar el protocolo de evaluación prefijado. Dadas las dificultades que suele presentar la recogida de información mediante autoinformes en personas mayores, se recogió la sugerencia de utilizar tarjetas con las categorías de respuesta impresas, para facilitar su comprensión y no inducir o deducir la respuesta (Montorio e Izal, 1999). La evaluación consistió en una entrevista individual realizada por licenciados en psicología con experiencia en el ámbito de la vejez y formación específica en el contenido de la entrevista.

\section{RESULTADOS}

La estructura de subescalas de contenidos de la WSOA-R fue generada racionalmente a partir de datos cualitativos y no empíricamente, por lo que primeramente se comprobó hasta qué punto los items se agrupan en los dominios de contenido que propone la escala. Para ello realizamos un Análisis Factorial, empleando Componentes Principales como método de extracción, en el que 20 factores tuvieron un autovalor superior a 1. Sin embargo, el gráfico de sedimentación mostraba un cambio en la pendiente entre los factores 5 y 6 , por lo que se retuvo una solución de 5 factores que explicaban el $57.1 \%$ de la varianza total. La matriz de componentes con estos cinco factores fue sometida a continuación a una rotación ortogonal (varimax). A partir de la matriz rotada decidimos eliminar aquellos items que no tuvieran saturaciones elevadas en ningún factor y los items complejos, con saturaciones elevadas en más de un factor. De este modo, se eliminaron aquellos items que no presentaron una saturación igual o superior a 0,50 en ninguno de los cinco factores retenidos y los items con saturaciones iguales o superiores a $\mathbf{0 , 5 0}$ en algún factor que además tuvieran saturaciones iguales o superiores a 0,40 en algún otro factor (Hair, Anderson, Tatham y Black, 1999). De este modo se retuvieron sólo 35 items, entre los que se encontraban 8 inicialmente asignados a la escala de preocupaciones sobre la Salud, 4 de Economía, 6 de Familia, 6 de Aspectos Sociales y 11 de Aspectos Personales. No se mantuvo ningún ítem de la escala de preocupaciones SocioPolíticas. En la Tabla 1 puede verse la matriz rotada, con las saturaciones de cada ítem en cada uno de los cinco factores tras la rotación ortogonal y la correlación entre cada ítem y su correspondiente subescala. 
Tabla 1. Estructura factorial de la Escala de Preocupaciones (EP)(rotación varimax) y correlación ítem-subescala

\begin{tabular}{|c|c|c|}
\hline & $\begin{array}{c}\text { saturaciones en } \\
\text { cada factor }\end{array}$ & $\begin{array}{c}\text { correlación ítem } \\
\text { sub-escala }\end{array}$ \\
\hline \multicolumn{3}{|l|}{ SALUD E INTEGRIDAD FÍSICA } \\
\hline Que entren en su casa y la destrocen* & 0,76 & 0,71 \\
\hline Tener una crisis nerviosa & 0,74 & 0,75 \\
\hline No poder levantarse de la cama por la mañana & 0,69 & 0,53 \\
\hline Tener que ir a una residencia u hospital* & 0,69 & 0,57 \\
\hline Que le asalten o roben por la calle* & 0,66 & 0,61 \\
\hline \multicolumn{3}{|l|}{ Que su marido/ mujer o un familiar cercano puedan tener una } \\
\hline enfermedad 0 accidente grave ${ }^{\star}$ & 0,65 & 0,70 \\
\hline Tener una enfermedad o accidente grave ${ }^{*}$ & 0,63 & 0,65 \\
\hline Tener problemas psicológicos graves* & 0,63 & 0,69 \\
\hline Tener que utilizar ayudas mecánicas (audífono, bastón...)* & 0,61 & 0,43 \\
\hline Deprimirse $^{*}$ & 0,60 & 0,68 \\
\hline Caerse y romperse algo & 0,58 & 0,56 \\
\hline No poder dormir por las noches* & 0,54 & 0,42 \\
\hline \multicolumn{3}{|l|}{ FAMILIA } \\
\hline Que sus hijos no tengan suerte & 0,82 & 0,80 \\
\hline Que sus hijos no sean felices & 0,77 & 0,72 \\
\hline Que sus hijos tengan problemas graves de salud & 0,76 & 0,72 \\
\hline Que sus hijos y nietos no tomen la decisiones adecuadas & 0,76 & 0,73 \\
\hline Que sus hijos no vivan en un mundo seguro & 0,71 & 0,64 \\
\hline Que sus hijos no sean capaces de valerse por sí mismos & 0,71 & 0,63 \\
\hline \multicolumn{3}{|l|}{ PÈRDIDA DE COMPETENCIAS PERSONALES } \\
\hline Tener que hacer nuevos amigos & 0,78 & 0,52 \\
\hline Tener que tomar decisiones sin ayuda & 0,67 & 0,63 \\
\hline No ser capaz de hacer nuevos amigos & 0,62 & 0,48 \\
\hline No poder tomar decisiones* & 0,61 & 0,52 \\
\hline Que sus amigos y familia no le visiten ${ }^{\star}$ & 0,54 & 0,40 \\
\hline No poder dejar sus asuntos en orden & 0,52 & 0,50 \\
\hline No poder mantenerse ocupado & 0,50 & 0,52 \\
\hline No poder hacer lo que quiera, como quiera y cuando quiera & 0,46 & 0,46 \\
\hline \multicolumn{3}{|l|}{ ECONOMÍA } \\
\hline Que su dinero no dure lo suficiente & 0,83 & 0,75 \\
\hline No poder manejar su propio dinero & 0,76 & 0,60 \\
\hline No poder pagar necesidades básicas* & 0,71 & 0,51 \\
\hline No tener el dinero que reserva para la jubilación & 0,69 & 0,57 \\
\hline \multicolumn{3}{|l|}{ SOCIO-AFECTIVO } \\
\hline Que la gente le vea poco interesante* & 0,87 & 0,70 \\
\hline Que la gente no tenga buena opinión de Vd. & 0,80 & 0,67 \\
\hline Que nadie quiera estar cerca de $\mathrm{Vd} .^{*}$ & 0,76 & 0,62 \\
\hline Tener aspecto de viejo* & 0,55 & 0,36 \\
\hline Que nadie le quiera* & 0,55 & 0,37 \\
\hline
\end{tabular}

Nota: * Ítem perteneciente a la WS original de 35 Items (Wisocki et al., 1986)

El primer factor explicó un $26,84 \%$ de la varianza (con un autovalor de 9,39) y agrupa items referidos a peligro físico (p.ej., «Que le asalten o roben por la calle») y a salud física y psicológica (p.ej.,
"Caerse y romperse algo»), por lo que lo hemos denominado Salud e Integridad Física. Incluye 12 items, todos en sentido positivo, por lo que el rango de puntuación es de 0 a 48 . La subescala pre- 
sentó una consistencia interna (alpha de Cronbach) muy buena (según la clasificación de De Vellis, 1991): 0,88. El segundo factor explicó un $9,13 \%$ de la varianza (autovalor de 3,19 ) y agrupa a 6 items, todos ellos relacionados con preocupaciones sobre el bienestar de la familia (p.ej., "Que sus hijos no sean felices»), por lo que hemos denominado al factor como Familia. El rango de puntuación para esta escala es de 0-24 y la consistencia interna fue muy buena (alpha de Cronbach $=0,89$ ). El tercer factor explicó un $8,11 \%$ de la varianza (autovalor de $2,84)$ y agrupa elementos relacionados a la preocupación por la posible pérdida de diferentes competencias personales (p.ej., «tener que hacer nuevos amigos» o "no poder tomar decisiones»), por lo que hemos etiquetado este factor como Competencias Personales. La escala la componen 8 items, lo que implica un rango de puntuación de 0-32, y presenta una consistencia interna aceptable $(0,78)$. El cuarto factor explicó un $7,03 \%$ de la varianza (autovalor de 2,46 ) y lo componen items referidos claramente a preocupaciones económicas, por lo que denominanos este factor como Economía. La escala la componen 4 items (rango $=0-16$ ) y presenta una consistencia interna aceptable $(0,79)$. Finalmente, el quinto factor explicó un $6,0 \%$ de la varianza (autovalor de 2,10 ) y en él tienen saturaciones elevadas items vinculados a la imagen externa o la opinión que los demás puedan tener de la persona, por lo que lo hemos llamado Socio-Afectivo. Incluye 5 items (rango $=0-20$ ) y presenta una consistencia interna aceptable $(0,75)$. Las puntuaciones medias (con las desviaciones típicas entre paréntesis) de las subescalas y de la puntuación total fueron las siguientes: Salud e Integridad Física, 11,2 (10,2); Familia, 6,7 (6,2); Pérdida de Competencias Personales, 3,4 (4,6); Economía, $2,1(3,5)$; aspectos Socio-afectivos, 1,7 $(2,8)$; Puntuación total, $24,6(19,4)$. Los items fueron ordenados aleatoriamente para componer una escala de evaluación de los contenidos de preocupación en las personas mayores. Esta escala se presenta en el Apéndice 1. La denominación final de la escala así configurada es Escala de Preocupaciones (EP).

Respecto a la comparación de la EP con la asignación racional de los items a cada escala de contenido en la versión original WSOA-R (Wisocki, 1994), los items de la escala de Economía y Familia se agrupan juntos en sendas escalas confirmando la agrupación racional de estos items en la escala original. Sin embargo, en este estudio los items de las escalas de Salud y de Aspectos Personales, y, en menor medida, los referidos a Aspectos Sociales, aparecen fuertemente entremezclados en las tres subescalas restantes. Es interesante comprobar que 16 de los 35 items a los que se ha llegado mediante la reducción por Análisis Factorial de la escala ampliada, se encontraban ya en la escala original de 35 items (Wisocki et al., 1986). Estos items se señalan con un asterisco en la Tabla 1.

A continuación, mediante análisis de correlación bivariados se analizó la relación de estas cinco dimensiones de contenido de la EP con las medidas de preocupación-rasgo, ansiedad-rasgo y gravedad de la depresión. Todas las correlaciones fueron positivas y significativas, con la excepción de la escala Socioafectiva, que no presentó correlaciones significativas con preocupación-rasgo ni con depresión. Las correlaciones entre las subescalas de contenidos fueron positivas y significativas, con la excepción de Socio-Afectivo con otras dos subescalas (Salud y Economía). Las correlaciones de Economía con otras subescalas son también bajas aunque alcancen la significación. En todo caso, dado que la escala proviene de una rotación ortogonal no eran de esperar fuertes relaciones entre las subescalas. Estos resultados pueden verse en 
la Tabla 2. Adicionalmente, para determinar la validez discriminante de la escala resultante se analizó la correlación de las puntuaciones de las subescalas y la puntuación total de la Escala de Preocupación con la obtenida en el Mini-Examen Cognoscitivo (MEC; Lobo et al., 1979), bajo la hipótesis de que no deberían ser significativas las correlaciones, como así ocurrió en todos los casos.

Con el fin de analizar si existían diferencias en los contenidos básicos de preocupación resumidos en las escalas retenidas en función de la presencia o no de una preocupación excesiva e indicativa de una posible patología, se realizaron comparaciones de medias mediante pruebas $\mathrm{T}$ para muestras independientes entre personas con niveles bajos de preocupación y personas que presentaban una preocupación excesiva (aunque no necesariamente TAG). El criterio para identificar a las personas con preocupación excesiva fue obtener una puntuación igual o superior a 50 en la escala que evalúa preocupación-rasgo, el PSWQ (Meyer et al., 1990). Brown et al. (1992), encontraron que todos los subgrupos con un trastorno de ansiedad diagnosticado (con la excepción de la fobia específica) presentaban una puntuación media en el PSWQ superior a esta puntuación. Este punto de corte divide a la muestra de este estudio en dos subgrupos: preocupación clínicamente no relevante $(n=85)$ y preocupación clínicamente relevante $(n=20)$. Se produjeron diferencias estadísticamente significativas en Salud e Integridad Física $(p<0,001)$, Familia $(p<0,01)$, Pérdida de Competencias Personales $(p<0,01)$ y Economía $(p<0,01)$. En la escala referida a aspectos Socio-afectivos no se produjeron diferencias estadísticamente significativas. Los resultados de las comparaciones de medias están resumidos en la Tabla 3 . Como puede verse en la tabla, las diferencias más marcadas se producen en la subescala de Salud e Integridad Física. Adicionalmente, realizamos pruebas $T$ entre estos mismos dos grupos (preocupación-rasgo clínicamente relevante o no) para cada uno de los 35 items incluidos en la escala. Todas las diferencias fueron estadísticamente significativas excepto en el caso de un ítem de Salud e Integridad Física («Tener que utilizar ayudas mecánicas»), 2 items de Familia ( $Q$ Que sus hijos y nietos no tomen las decisiones adecuadas" y "Que sus hijos no sean capaces de valerse por sí mismos»), 1 ítem de Pérdida de Competencias Personales ( No poder hacer lo que quiera, como quiera y cuando quiera») y 4 de los 5 items de

Tabla 2. Matriz de correlaciones entre las diferentes variables

\begin{tabular}{|c|c|c|c|c|c|c|c|c|c|}
\hline & PSWQ & STAI-R & GDS & Salud & Familia & $\begin{array}{c}\text { Compe- } \\
\text { tencias }\end{array}$ & $\begin{array}{c}\text { Econo- } \\
\text { mía }\end{array}$ & $\begin{array}{c}\text { Socio } \\
\text { afectivo }\end{array}$ & $\begin{array}{c}\text { Total } \\
\text { EP }\end{array}$ \\
\hline STAI-R & $0,79^{\star \star}$ & & & & & & & & \\
\hline GDS & $0,76^{\star \star}$ & $0,78^{\star \star}$ & & & & & & & \\
\hline Salud & $0,58^{* *}$ & $0,49^{\star \star}$ & $0,54^{\star \star}$ & & & & & & \\
\hline Familia & $0,42^{\star \star}$ & $0,41^{\star \star}$ & $0,35^{\star *}$ & $0,41 * *$ & & & & & \\
\hline Competencias & $0.53^{\star \star}$ & $0,58^{\star \star}$ & $0,52^{\star \star}$ & $0,41^{\star *}$ & $0,43^{\star \star}$ & & & & \\
\hline Economía & $0,39^{\star \star}$ & $0,38^{\star \star}$ & $0,33^{\star \star}$ & $0,22^{\star}$ & $0,22^{*}$ & $0,39 *$ * & & & \\
\hline Socioafectivo & 0,12 & $0,27^{\star *}$ & 0,11 & 0,16 & $0,30^{\star \star}$ & $0,29 * \star$ & 0,11 & & \\
\hline Total EP & $0,66^{\star \star}$ & $0,79^{\star \star}$ & $0,60^{\star *}$ & $0,86^{\star *}$ & $0,74^{\star \star}$ & $0,73^{\star \star}$ & $0,45^{* *}$ & $0,45^{\star \star}$ & \\
\hline MEC & $-0,07$ & $-0,05$ & $-0,02$ & $-0,15$ & 0,01 & $-0,08$ & $-0,07$ & 0,09 & $-0,10$ \\
\hline
\end{tabular}

Nota: * $p<0,05 ;{ }^{*}{ }^{*} p<0,01$ 
Socio-Afectivo (la excepción es «Tener aspecto de persona mayor").

\section{DISCUSIÓN}

El principal objetivo de este trabajo era analizar los contenidos de preocupación entre las personas mayores y, adicionalmente, comprobar si existen diferencias en esos dominios en función del nivel de preocupación (clínicamente relevante o no). De acuerdo al primer objetivo, mediante la reducción por criterios factoriales de un amplio listado de preocupaciones específicas se obtienen finalmente cinco dimensiones de preocupación, que hemos denominado Salud e Integridad Física, Familia, Competencias Personales, Economía y Socio-Afectivo. Estas cinco dimensiones parecen resumir razonablemente bien los aspectos principales de la vida de las personas mayores que son susceptibles de producir una amenaza a su vida cotidiana y por lo tanto de iniciar episodios de preocupación, con independencia de la frecuencia y gravedad con la que una persona tienda a preocuparse, es decir, podrían ser los ejes básicos de los aspectos en los que se enfoca la preocupación general en esta población. Las subescalas que evalúan las cinco dimensiones presentan, por otro lado, correlaciones positivas y significativas con medidas conceptualmente relacionadas como la ansiedad-rasgo, la preocupación-rasgo y la depresión y permiten diferenciar entre personas con un nivel de preocupación clínicamente relevante o no. La excepción es el dominio Socio-Afectivo, que sólo correlaciona significativamente con la ansiedad-rasgo y no distingue entre personas con niveles clínicos o no de preocupación-rasgo. Aunque esto podría ser un motivo para eliminar los cinco items de esta subescala, se ha decidido retenerla dado que el objetivo era obtener una medida no necesariamente clínica de los contenidos de preocupación.

Las dimensiones finalmente extraídas concuerdan de manera global con los resultados de estudios previos que indican el incremento de la relevancia de estos dominios de preocupación según las personas van envejeciendo, especialmente de las preocupaciones sobre la salud y la familia (Diefenbach et al., 2001; Doucet, Ladouceur, Freeston y Dugas, 1998; Montorio et al., 2003; Person y Borkovec 1995; Wisocki, 1994). La diferencia más llamativa entre las preocupaciones que han aparecido como las más relevantes en este estudio en comparación con estudios realizados con población de menos edad se presenta en la importancia de las preocupaciones familiares y de las preocupaciones sobre la posibilidad de llegar a perder la independencia funcional. Las situaciones

Tabla 3. Resultados de la comparación de medias de contenidos en función del nivel de preocupación (Clínica o Normal), según PSWQ $\leq 50$

\begin{tabular}{lccl}
\hline \multicolumn{1}{c}{ Subescala } & $\begin{array}{c}\text { No clínico } \\
(\mathrm{n}=80)\end{array}$ & $\begin{array}{c}\text { Clínico } \\
(\mathrm{n}=25)\end{array}$ & \multicolumn{1}{c}{$t_{103}$} \\
\hline Salud (12 items) (Rango: 0-48) & $8,9(7,7)$ & $21,5(13,6)$ & $5,5^{\star \star *}$ \\
Familia (6 items) (0-24) & $6,0(6,0)$ & $10,1(6,3)$ & $2,7^{\star *}$ \\
Pérdida de competencias personales (8 items) $(0-32)$ & $2,5(3,3)$ & $7,8(7,1)$ & $3,2^{\star *}$ \\
Economía (4 items) (0-16) & $1,4(2,6)$ & $5,1(5,1)$ & $3,0^{\star \star}$ \\
Socio-afectivo (5 items) (0-20) & $1,6(2,9)$ & $2,0(2,5)$ & 0,6 \\
\hline Total (35 items) (0-140) & $20,0(16,9)$ & $43,9(17,5)$ & $5,6^{\star \star *}$ \\
\hline
\end{tabular}

Nota: Los datos para cada grupo son medias y los datos entre paréntesis desviaciones típicas.

${ }^{\star \star} p<0,01 ;{ }^{\star \star \star} p<0,001$ 
vitales que parecen afectar a adultos jóvenes susceptibles de producir preocupación se asocian, por el contrario, principalmente a los aspectos socioevaluativos (p.ej., Eysenck, 1992), aunque también se ha señalado frecuentemente la relevancia de un segundo factor relacionado con las amenazas a la salud y a la integridad física. El último factor que emerge en este trabajo podría cubrir, aunque lógicamente adaptado a las circunstancias vitales de las personas mayores, esta dimensión socioevaluativa, reflejando la existencia entre las personas mayores de un dominio de preocupación asociado a los aspectos socioevaluativos. Sin embargo, no sólo es el menos importante, sino que además es el único en el que no hemos encontrado diferencias en función del nivel de preocupación-rasgo, lo que avalaría la menor relevancia de este dominio entre las personas mayores. Este cambio en la fenomenología de la preocupación puede asociarse a los cambios y adaptaciones que implica el envejecimiento normal (Borkovec, 1988), y así explicarse desde factores motivacionales asociados al ciclo evolutivo. Cuando una persona envejece, normalmente ya ha satisfecho la mayoría de las metas sociales (encontrar trabajo estable, formar una familia, etc.) o percibe que ya no tiene tiempo para alcanzarlas, por lo que es normal que ocurra un cambio en el enfoque de sus motivaciones y de las situaciones que considera que pueden amenazar o afectar a su satisfacción vital y que, por lo tanto, podrían generar preocupación. Por otra parte, existe una amplia evidencia en la literatura gerontológica de que a medida que las personas envejecen la familia gana en importancia, hecho que recientemente se ha explicado desde la Teoría de la Selectividad Socioemocional de Carstensen (1991, 1993). Esta teoría propone que según las personas van percibiendo su tiempo como limitado se van especializando en maxi- mizar las opciones de experimentar emociones positivas, lo que en el ámbito social se expresaría en centrarse en las relaciones cercanas e íntimas (familia y amigos íntimos), que reducen al máximo el coste de obtener relaciones emocionalmente significativas y positivas (Montorio et al., 2003). Respecto a los otros contenidos de preocupación que emergen en este estudio como relevantes en la vejez, la salud, la economía y la posible pérdida de competencias personales, existe evidencia empírica de que las personas mayores puede ser especialmente susceptibles a sentir ansiedad ante situaciones que disminuyan el control que tienen sobre los acontecimientos de sus vidas (Rodin y Timko, 1992). Las amenazas a la salud física, al mantenimiento del estatus económico o a la independencia funcional incrementan su posibilidad de ocurrencia con el envejecimiento y son situaciones que amenazan directamente la capacidad de control que una persona puede ejercer sobre los sucesos de su vida, lo que producirá, además, que este tipo de preocupaciones tenga un mayor impacto emocional entre las personas mayores que en otros grupos de edad (Heckhausen y Schulz, 1995).

En esta misma línea, los análisis en función del nivel de gravedad de la preocupación ofrecen diferencias estadísticamente significativas en todos los dominios de contenido, excepto en el Socio-Afectivo. En el caso de las preocupaciones por la salud, ya otros estudios han indicado la importancia de este tipo de preocupación en la vejez y su capacidad de discriminación sobre las alteraciones emocionales y el TAG en esta edad (p.ej., Diefenbach, Stanley y Beck, 2001; Montorio et al., 2003; Nuevo, Montorio, Márquez, Izal y Losada, en prensa; Person y Borkovec, 1995; Wisocki, 1994). Como se acaba de indicar, las amenazas al control personal sobre los sucesos de la propia vida que suponen en la vejez los proble- 
mas de salud, así como la perdida de la independencia funcional, podrían implicar un mayor impacto emocional de estas preocupaciones (Schulz y Heckhausen, 1996), lo que daría cuenta de la mayor preocupación sobre estos contenidos entre las personas con preocupación clínicamente relevante. En el caso de las preocupaciones sobre la familia estas diferencias podrían deberse a las amenazas que los problemas en la familia suponen potencialmente para la necesidad de las personas mayores en general de optimizar sus posibilidades de implicarse en relaciones afectivas positivas (Carstensen, 1991, 1993). Se ha propuesto la posibilidad de que esta necesidad sea un efecto de la percepción del tiempo como progresivamente limitado que acompaña usualmente al envejecimiento (Carstensen, Isaacowitz y Charles, 1999; Siguán, 1993), ya que se ha encontrado una tendencia muy similar en personas con una enfermedad terminal al comparar personas mayores con adultos jóvenes sanos, otros diagnosticados de SIDA aunque sin síntomas y con casos sintomáticos de SIDA (Carstensen y Fredrickson, 1998).

$\mathrm{La}$ escala finalmente propuesta (EP, apéndice 1), compuesta por 35 items distribuidos al azar y agrupados en las cinco dimensiones indicadas, podría ser un buen método de evaluar los contenidos de preocupación en las personas mayores. Las condiciones de aleatorización de la muestra y la amplitud del listado inicial de items permiten proponer este instrumento como una buena medida de los contenidos de la preocupación normal en población mayor española.

Aún son precisos, sin embargo, futuros estudios que analicen en profundidad las propiedades psicométricas de esta escala. Las conclusiones sobre la capacidad de las subescalas para distinguir entre personas con niveles de preocupación clínicamente relevantes o no, están fuertemente limitadas por el hecho de que se ha utilizado la misma muestra para la reducción factorial de la escala y para esas comparaciones. Además, la muestra de análogos clínicos es reducida y poco específica respecto al tipo de posible patología vinculada a la preocupación clínicamente relevante. Es precisa entonces una validación externa de las propiedades psicométricas de la escala propuesta, con amplias muestras de personas mayores tanto sin trastorno emocional como con diferentes tipos de trastornos de ansiedad o del estado de ánimo. Por otra parte, aunque el listado inicial de items es suficientemente amplio y fue generado a partir de un trabajo cualitativo con grupos de discusión de personas mayores, no deja de ser una escala cerrada, por lo que no se ajustaría a la recomendación de Dugas et al. (1995) de utilizar medidas totalmente abiertas en la fase inicial de construcción de un instrumento de contenidos de preocupación. Sin embargo, se incluyeron preguntas abiertas antes y después de la aplicación de la WSOA-R y no apareció ningún contenido que no pudiera ser asimilado a los 83 incluidos en la escala, de manera que este problema no parece haber afectado en exceso a los resultados.

\section{REFERENCIAS BIBLIOGRÁFICAS}

Asociación Psiquiátrica Americana (2001). Manual diagnóstico y estadístico de los trastornos mentales ( $4^{a}$ edición, texto revisado) (DSM-IV-TR). Barcelona, Masson (original de 2000).

Arnkoff, C.B., y Glass, D.B. (1989). Behavioral assessment of social anxiety and social phobia. Clinical Psychology Review, 9, 75-90.

Beck, J.G., Stanley, M.A., y Zebb, B.J. (1995). Psychometric properties of the Penn State Worry Questionnaire in older adults. Journal of Clinical Geropsychology, 1, 33-42.

Borkovec, T.D. (1988). Comments on «Worry as a phenomenon relevant to the elderly». Behavior Therapy, 19, 381-383. 
Bower, G.H. (1981). Mood and memory. American Psychologist, 36, 129-148.

Brown, T.A., Antony, M.M., y Barlow, D.H. (1992). Psychometric properties of the Penn State Worry Questionnaire in a clinical anxiety disorders sample. Behaviour Research and Therapy, 30, 33-37.

Brink, T.L., Yesavage, J.A., Lum, O., Heersema, P., Adey, M., y Rose (1982). Screening tests for geriatric depression. Clinical Gerontologist, 1, 37-44.

Carstensen, L.L. (1991). Selectivity theory: Social activity in life-span context. Annual Review of Gerontology \& Geriatrics, 11 , 195-217.

Carstensen, L.L. (1993). Motivation for social contacts across the life span: A theory of socioemotional selectivity. Nebraska Symposium on Motivation, 40, 209-254.

Carstensen, L.L., y Fredrickson, B.F. (1998). Socioemotional selectivity in healthy older people and younger people living with the human immunodeficiency virus: The centrality of emotion when the future is constrained. Health Psychology, 17, 1-10.

Carstensen, L.L., Isaacowitz, D.M., y Charles, S.T. (1999). Taking time seriously: A theory of socioemotional selectivity. American Psychologist, 54, 165-181.

Craske, M.G., Rapee, R., Jackel, L., y Barlow, D.H. (1989). Qualitative dimensions of worry in DSM-III-R generalized anxiety disorder subjects and nonaxious controls. Behaviour Research and Therapy, 27, 397 402.

De Vellis, R.F.C. (1991). Scale development : Theory and applications. Newsbury Park, CA, Sage.

Diefenbach, G.J., Stanley, M.A., y Beck, J.G. (2001). Worry content reported by older adults with and without generalized anxiety disorder. Aging and Mental Health, 5, 269-274.

Doucet, C., Ladouceur, R., Freeston, M.H., y Dugas, M.J. (1998). Thèmes d'inquiétudes et tendance à s'inquiéter chez les aînes. Canadian Journal on Aging, 17, 361-371.

Dugas, M.J., Freeston, M.H., Doucet, C., Lachance, S., y Ladouceur, R. (1995). Structured versus free-recall measures: Effect on report of worry themes. Personality and Individual Differences, 18, 355-362.
Dugas, M.J., Freeston, M., Ladouceur, R., Rheaume, J., Provencher, M., y Boisvert, J.M. (1998). Worry themes in primary GAD, secondary GAD and other anxiety disorders. Journal of Anxiety Disorders, 12, 253-261.

Eysenck, M.W. (1984). Anxiety and the worry process. Bulletin of the Psychonomic Society, 22, 545-548.

Eysenck, M.W. (1992). Anxiety. The cognitive perspective. Hove, LEA.

Folstein, M.F., Folstein, S.E., y McHugh, P.R. (1975). Mini-mental state: a practical method for grading the cognitive state of patients for the clinician. Journal of Psychiatry Research, 12, 189-198.

Fresco, D.M., Heimberg, R.G., Mennin, D.S., y Turk, C.L. (2002). Confirmatory factor analysis of the Penn State Worry Questionnaire. Behaviour Research and Therapy, 40, 313-323.

Hair, J.F., Anderson, R.E., Tatham, R.L., y Black, W.C. (1999). Análisis Multivariante ( $5^{q}$ edición). Madrid: Prentice Hall.

Heckhausen, J., y Schulz, R. (1995). A lifespan theory of control. Psychological Review, 102, 284-304.

Izal, M., y Montorio, I. (1996). Adaptación en nuestro medio de la Escala de Depresión Geriátrica (GDS) en distintos subgrupos: residentes en la comunidad y asistentes a Hospitales de Día. Revista de Gerontología, 6, 329-338.

Ladouceur, R., Freeston, M.H., Dumont, J., Letarte, H., Rhéaume, J., Gagnon, F., y Thibodeau, N. (1992). Penn State Worry Questionnaire: Validity and reliability of a french translation. Canadian Psychology, 33, 236.

Lobo, A., Ezquerra, J., Gómez, F.B., Sala J.M., y Seva, A. (1979). El Mini-Examen Cognoscitivo. Un test sencillo y práctico para detectar alteraciones intelectuales en pacientes médicos. Actas Luso-Españolas de Neurología Psiquiátrica y Científica, 7 , 189-202.

Meyer, T.J., Miller, M.L., Metzger, R.L., y Borkovec, T.D. (1990). Development and validation of the Penn State Worry Questionnaire. Behaviour Research and Therapy, 28, 487-495.

Montorio, I., e Izal, M. (1996). The Geriatric Depression Scale: a review of its develop- 
ment and utility. International Psychogeriatrics, $8,103-112$.

Montorio, I., e Izal, M. (Eds.) (1999). Intervención psicológica en la vejez. Aplicaciones en el ámbito clínico y de la salud. Síntesis: Madrid.

Montorio, I., Nuevo, R., Izal, M., y Losada, A. (1998). La Escala de Preocupaciones para Personas Mayores: utilidad para la evaluación de la ansiedad generalizada. Comunicación presentada en el V Congreso Nacional de Psicología Conductual, Vitoria.

Montorio, I., Nuevo, R., Márquez, M., Izal, M., y Losada, A. (2003). Characterization of worry according to the severity of anxiety. Aging and Mental Health, 7, 334-341.

Nuevo, R., Montorio, I., Márquez, M., Izal, M., y Losada, A. (en prensa). Análisis del fenómeno de la preocupación en personas mayores. Revista Internacional de Psicología Clínica y de la Salud.

Nuevo, R., Montorio, I., y Ruiz, M.A. (2002). Aplicabilidad del Inventario de Preocupación de Pensilvania (PSWQ) a población de edad avanzada. Ansiedad y Estrés, 8, 157-172.

Person, D.C., y Borkovec, T.D. (1995, Agosto). Anxiety disorders among the elderly: Patterns and issues. Comunicación presentada en el 103 Annual Meeting of the American Psychological Association, New York.

Powers, C.B., Wisocki, P.A., y Whitbourne, S.K. (1992). Age differences and correlates of worrying in young and elderly adults. The Gerontologist, 32, 82-88.

Pratt, P., Tallis, F., y Eysenck, M.W. (1997). Information-processing, storage characteristics and worry. Behaviour Research and Therapy, 35, 1015-1023.

Rodin, J., y Timko, C. (1992). Sense of control, aging, and health. In M.G. Ory y R.P. Abeles, Aging, health and behaviour (pp. 174-206). Thousand Oaks: Sage.

Sanderson; W.C., y Barlow, D.H. (1990). A description of patients diagnosed with DSM-III-R Generalized Anxiety Disorder. The Journal of Nervous and Mental Disease, 178, 588-591.

Schaie, K.W. (1994). The course of adult intellectual development. American Psychologist, 4, 304-313.
Schulz, R., y Heckhausen, J. (1996). A lifespan model of successful aging. American Psychologist, 51, 702-709.

Shadick, R.N., Roemer, L., Hopkins, M.B., y Borkovec, T.D. (1991). The nature of worrisome thoughts. Comunicación presentada en la reunión anual de la Association for Advancement of Behavior Therapy, Nueva York.

Siguán, M. (1993). Tres reflexiones sobre la temporalidad humana. En V. Pelechano (Comp.), Psicología, mitopsicología y postpsicología (pp. 43-76). Valencia: Promolibro.

Skarborn, M., y Nicki, R. (1996). Worry among canadian seniors. International Journal of Aging and Human Development, 43, 169-178.

Spielberger, C.D., Gorsuch, R.L., y Lushene, R.E. (1970). STAI manual for the StateTrait Anxiety Inventory. Palo Alto, Consulting Psychologist Press (versión española, TEA, 1982).

Starcevic, V. (1995). Pathological worry in major depression: A preliminary report. Behaviour Research and Therapy, 33, 55-56.

Tallis, F., Eysenck, M., y Mathews, A. (1991). The role of temporal perspective and egorelevance in the activation of worry structures. Personality and Individual Differences, 12, 909-915.

Tallis, F., Eysenck, M., y Mathews, A. (1992). A questionnaire for the measurement of nonpathological worry. Personality and Individual Differences, 13, 161-168.

Wells, A. (1994). A multidimensional measure of worry: Development and preliminary validation of the Anxious Thoughts Inventory. Anxiety, Stress and Coping, 6, 289299.

Wisocki, P.A. (1988). Worry as a phenomenon relevant to the elderly. Behavior Therapy, 19, 369-379.

Wisocki, P.A. (1994). The experience of worry among the elderly. En G.C.L. Davey y F. Tallis, Worrying. Perspectives on theory, assessment and treatment (pp. 247-261). Chichester: Wiley.

Wisocki, P.A., Handen, B., y Morse, C.K. (1986). The Worry Scale as a measure of anxiety among home bound and community active elderly. The Behavior Therapist, 5, 91-95. 


\section{APÉNDICE}

\section{ESCALA DE PREOCUPACIONES (EP)}

A continuación verá una lista de problemas concretos por los que muchas personas mayores se preocupan. Por favor, léalos y señale la opción que mejor describa con qué frecuencia se ha preocu- pado usted por cada uno de ellos en las últimas dos semanas. Debe indicar con qué frecuencia ha pensado realmente en ello, no si le preocupa o le preocuparía que ese problema ocurriese.

\begin{tabular}{|c|c|c|c|c|c|}
\hline Le preocupa & Nunca & $\begin{array}{l}\text { Rara- } \\
\text { mente }\end{array}$ & $\begin{array}{c}\text { Algunos } \\
\text { Veces }\end{array}$ & $\begin{array}{c}\text { Frecuen- } \\
\text { mente }\end{array}$ & $\begin{array}{c}\text { Casi } \\
\text { siempre }\end{array}$ \\
\hline \multicolumn{6}{|l|}{ 1. No poder pagar necesidades básicas } \\
\hline \multicolumn{6}{|l|}{ 2. Que le asalten o roben por la calle } \\
\hline \multicolumn{6}{|l|}{ 3. Caerse y romperse algo } \\
\hline \multicolumn{6}{|l|}{ 4. Que su dinero no dure lo suficiente } \\
\hline \multicolumn{6}{|l|}{$\begin{array}{l}\text { 5. Que sus hijos y nietos no tomen la decisiones } \\
\text { adecuadas }\end{array}$} \\
\hline \multicolumn{6}{|l|}{ 6. Tener una crisis nerviosa } \\
\hline \multicolumn{6}{|l|}{ 7. No ser capaz de hacer nuevos amigos } \\
\hline \multicolumn{6}{|l|}{ 8. Tener que ir a una residencia u hospital } \\
\hline \multicolumn{6}{|l|}{ 9. No poder manejar su propio dinero } \\
\hline \multicolumn{6}{|l|}{ 10. Tener problemas psicológicos graves } \\
\hline \multicolumn{6}{|l|}{ 11. Tener aspecto de viejo } \\
\hline \multicolumn{6}{|l|}{$\begin{array}{l}\text { 12. Que su marido/ mujer o un familiar cercano } \\
\text { puedan tener una enfermedad o accidente grave }\end{array}$} \\
\hline \multicolumn{6}{|l|}{ 13. Que la gente no tenga buena opinión de Vd. } \\
\hline \multicolumn{6}{|l|}{ 14. Deprimirse } \\
\hline \multicolumn{6}{|l|}{ 15. No tener el dinero que reserva para la jubilación } \\
\hline \multicolumn{6}{|l|}{ 16. No poder dormir por las noches } \\
\hline \multicolumn{6}{|l|}{ 17. Que sus hijos no tengan suerte } \\
\hline \multicolumn{6}{|l|}{ 18. No poder tomar decisiones } \\
\hline \multicolumn{6}{|l|}{ 19. Que sus hijos tengan problemas graves de salud } \\
\hline \multicolumn{6}{|l|}{ 20. Que entren en su casa y la destrocen } \\
\hline \multicolumn{6}{|l|}{ 21. Que sus hijos no vivan en un mundo seguro } \\
\hline \multicolumn{6}{|l|}{$\begin{array}{l}\text { 22. No poder hacer lo que quiera, como quiera y } \\
\text { cuando quiera }\end{array}$} \\
\hline \multicolumn{6}{|l|}{ 23. Tener que hacer nuevos amigos } \\
\hline \multicolumn{6}{|l|}{ 24. Tener que tomar decisiones sin ayuda } \\
\hline \multicolumn{6}{|l|}{ 25. No poder levantarse de la cama por la mañana } \\
\hline \multicolumn{6}{|l|}{ 26. Que sus hijos no sean felices } \\
\hline \multicolumn{6}{|l|}{ 27. No poder dejar sus asuntos en orden } \\
\hline \multicolumn{6}{|l|}{ 28. Que nadie le quiera } \\
\hline 29. No poder mantenerse ocupado & & & & & \\
\hline $\begin{array}{l}\text { 30. Que sus hijos no sean capaces de valerse por } \\
\text { sí mismos }\end{array}$ & & & & & \\
\hline 31. Que la gente le vea poco interesante & & & & & \\
\hline $\begin{array}{l}\text { 32. Tener que utilizar ayudas mecánicas (audífo- } \\
\text { no, bastón...) }\end{array}$ & & & & & \\
\hline 33. Que nadie quiera estar cerca de Vd. & & & & & \\
\hline 34. Tener una enfermedad o accidente grave & & & & & \\
\hline 35. Que sus amigos y familia no le visiten & & & & & \\
\hline
\end{tabular}


ITEMS POR SUBESCALAS

Salud: $2,3,6,8,10,12,14,16,20,25,32$ y 34 .

Familia: 5, 17, 19, 21, 26 y 30.

Pérdida de Competencias personales: 7, 18, 22, 23, 24, 27, 29 у 35.

Economía: 1, 4, 9 y 15.

Socio-afectiva: 11, 13, 28, 31 y 33 . 